\title{
Spontaneous Chiral Symmetry Breaking and Entropy Production in a Closed System
}

\author{
Dilip Kondepudi ${ }^{1, *}$ and Zachary Mundy ${ }^{2}$ \\ 1 Department of Chemistry, Wake Forest University, Winston-Salem, NC 27109, USA \\ 2 BASF, 100 Park Avenue, Florham Park, NJ 07932, USA; zachary.mundy@basf.com \\ * Correspondence: dilip@wfu.edu
}

Received: 2 March 2020; Accepted: 14 April 2020; Published: 6 May 2020

\begin{abstract}
In this short article, we present a study of theoretical model of a photochemically driven, closed chemical system in which spontaneous chiral symmetry breaking occurs. By making all the steps in the reaction elementary reaction steps, we obtained the rate of entropy production in the system and studied its behavior below and above the transition point. Our results show that the transition is similar to a second-order phase transition with rate of entropy production taking the place of entropy and the radiation intensity taking the place of the critical parameter: the steady-state entropy production, when plotted against the incident radiation intensity, has a change in its slope at the critical point. Above the critical intensity, the slope decreases, showing that asymmetric states have lower entropy than the symmetric state.
\end{abstract}

Keywords: chiral symmetry breaking; entropy production; closed systems; nonequilibrium; dissipative structures

\section{Introduction}

Modern thermodynamics, formulated in the 20th century by Onsager [1], De Donder [2], Prigogine [3,4], and others, introduced a critical concept lacking in its classical formulation: rate of entropy change and its relationship to irreversible processes. Classical thermodynamics was concerned with functions of state, such as energy and entropy, and their change from one equilibrium state to another. Absent from this theory of states is consideration of the rates of processes. Changes in entropy for infinitely slow reversible processes are calculated using the relation $\mathrm{dS}=\mathrm{dQ} / \mathrm{T}$, (in which $\mathrm{dS}$ is the change in entropy, $\mathrm{T}$ is the temperature in Kelvin, and $\mathrm{dQ}$ is the heat exchanged between a system and its exterior). However, for changes that take place in a finite time due to irreversible processes, the theory does not specify a way of calculating the entropy change; it is only stated that $d S>d Q / T$. Modern thermodynamics is a theory of processes in which thermodynamic forces and the flows they drive are identified and the rate of entropy production is expressed in terms of these thermodynamic forces and flows $[5,6]$. More specifically, the rate of entropy production per unit volume, $\sigma$, is expressed in terms of the forces and flows as

$$
\sigma=\frac{d s}{d t}=\sum_{k} F_{k} J_{k}
$$

in which $s$ is the entropy density, $F_{k}$ are the thermodynamic forces and $J_{k}$ are thermodynamic flows. A temperature gradient, for example, is the thermodynamic force, $F_{k}$, that drives thermodynamic flow, $J_{k}$, of heat current. The force that drives chemical reactions has been identified as affinity $[2,5,6]$ and the corresponding flow is the rate of conversion form reactants to products. This flow is expressed as the time derivative $\mathrm{d} \xi / \mathrm{dt}(\mathrm{mol} / \mathrm{s})$ of the extent of reaction $\xi[5,6]$. For an elementary chemical reaction step, 
the rate of entropy production can be written in terms of the forward reaction rate, $R_{f}$, and the reverse reaction rate, $R_{r}[6]$

$$
\sigma=R\left(R_{f}-R_{r}\right) \ln \left(\frac{R_{f}}{R_{r}}\right)
$$

in which $R$ is the gas constant. The total entropy production for a sequence of reactions is the sum of entropy productions of each reaction (indexed by $k$ ) [6]

$$
\sigma=R \sum_{k}\left(R_{k f}-R_{k r}\right) \ln \left(\frac{R_{k f}}{R_{k r}}\right)
$$

We note that calculations of entropy production using the above formulas require that the reverse reaction rates, $R_{r}$, are non-zero. In considering kinetic equations of a chemical system, often the reverse rates have high reaction barriers and, correspondingly, very low reaction rates, and are assumed to be "zero" because they are negligible compared to the forward rates. Low reverse reaction rates keep the system from evolving to equilibrium state. In the model we will present below, the system is driven far from equilibrium by an inflow of radiation. In the absence of radiation, our system evolves to equilibrium - there are no very high reaction barriers to keep it from reaching its equilibrium state.

It is well known that nonequilibrium chemical systems can undergo spontaneous symmetry-breaking transitions to organized structures called dissipative structures [6,7]. The large class of dissipative structures includes spatial patterns, chemical clocks, and structures with chiral asymmetry. These structures arise when a nonequilibrium system becomes unstable and undergoes a transition to new organized state. The model we present below shows a chiral symmetry breaking transition to an asymmetric state as the intensity of radiation increases.

The study of spontaneous chiral symmetry breaking in chemical systems has a long history, starting in the 1950s with the first model by Frank [8]. In the 1970s, when the theory of dissipative structures was developed, instability, bifurcation, and spontaneous symmetry breaking in non-equilibrium systems became the foundation for the study of chiral asymmetry we see in nature [4,7,9-11]. A general theory of chiral symmetry breaking in chemical systems, independent of the details of the chemical kinetics, was formulated, and it was used to study the sensitivity of chiral symmetry breaking systems to small chiral influences [11-13]. Using this theory, it was possible to calculate the time scales needed for a chiral-symmetry-breaking chemical system to be influenced by the chiral asymmetry of electro-weak interaction in molecules [14], and it was found that this timescale is of the order to $10^{4}$ years [15]. These results show that a lot of interesting and important general conclusions can be arrived at through using theoretical models $[16,17]$. Along these lines, we investigate the thermodynamic aspects of systems that spontaneously break chiral symmetry, using a model presented below.

Using nonequilibrium thermodynamics, we analyze the behavior of entropy production, $\sigma$, for a reaction scheme that consists of a photochemically driven closed system (that has no flow of matter). The incident radiation drives a generation-decomposition cycle of chiral molecules. At the critical intensity (above which the system breaks chiral symmetry), the slope of the rate of entropy production, $\sigma$, changes just as entropy does in a second-order phase transition. We have reported a similar result in a flow system with an inflow of reactants and outflow of products [18]. There is a basic difference, however: in our previous study, the slope of $\sigma$ increased, but in our current study we find that the slope decreased, although in both cases the behavior of $\sigma$ is similar to that in a second-order phase transition.

\section{Materials and Methods}

All reactions in our scheme are reversible so that the system can reach a state of chemical equilibrium. At equilibrium, the rate of entropy production is zero. The reaction scheme of this photochemically driven closed system is shown in Table 1. It is assumed to take place in a homogeneous aqueous phase in which radiation is incident. For a photochemical reaction, the intensity of a narrow band of wavelength is the relevant intensity, therefore it will have a low value compared to a typical 
intensity of blackbody radiation that includes all wavelengths-for example, radiation from the sun. An achiral molecule, $\mathrm{T}$, is photochemically activated to an excited species or a more reactive isomer, $\mathrm{TE}$, as shown in reaction (R1); II is the intensity of radiation of the exciting wavelength. TE can radiate its excitation energy and return to the unexcited state, $\mathrm{T}(\mathrm{R} 1 \mathrm{a})$, through various processes. During the transition of TE to $\mathrm{T}$, the emitted radiation undergoes scattering and thermalizes to the temperature of the system. (R1a) represents all the processes that keep TE and T at equilibrium (including thermal radiation [6]) in the absence of external radiation. In writing the reaction rates for $\mathrm{T}$ and $\mathrm{TE}$, we only need to include additional term in the forward reaction that includes II and the all reverse reactions, TE to T, combined into one reaction rate. The excited species TE can also react with an achiral molecule $\mathrm{S}$ to form a chiral species, $\mathrm{X}_{\mathrm{L}}$ or $\mathrm{X}_{\mathrm{D}}$ (R2 and R3). The set of reactions $\mathrm{R} 4 \mathrm{a}$ and $\mathrm{R} 4 \mathrm{~b}$ are elementary steps of an autocatalytic reaction for $X_{L}$ with the intermediate species $S_{L}$; similar reaction steps result in the autocatalytic production of $X_{D}$, as shown in R5a and R5b. Reaction steps R6, R7 and R8 show reactions through which the species decompose into starting material $S$ and $T$. The scheme explicitly has all the steps needed for the system to reach chemical equilibrium in the absence of radiation.

Table 1. A model photochemically driven reaction scheme in a closed system. The table lists all the elementary steps in the model. The excitation of $\mathrm{T}$ to the state TE drives the reaction that generate chiral species $X_{L}$ and $X_{D}$. Reactions $R 4 a, R 4 b, R 5 a, R 5 b$ are the autocatalytic steps for $X_{L}$ and $X_{D}$. Autocatalysis and reaction $\mathrm{R} 6$ result in spontaneous breaking of chiral symmetry when the intensity of radiation, $\mathrm{II}$, is above a critical intensity, $\mathrm{II}_{\mathrm{C}}$.

\begin{tabular}{cc}
\hline Chemical Reactions & Number \\
\hline $\mathrm{S}+\mathrm{II} \rightleftharpoons \mathrm{TE}$ & $(\mathrm{R} 1)$ \\
$\mathrm{T} \rightleftharpoons \mathrm{TE}$ & $(\mathrm{R} 1 \mathrm{a})$ \\
$\mathrm{S}+\mathrm{TE} \rightleftharpoons \mathrm{X}_{\mathrm{L}}$ & $(\mathrm{R} 2)$ \\
$\mathrm{S}+\mathrm{TE} \rightleftharpoons \mathrm{X}_{\mathrm{D}}$ & $(\mathrm{R} 3)$ \\
$\mathrm{S}+\mathrm{X}_{\mathrm{L}} \rightleftharpoons \mathrm{S}_{\mathrm{L}}$ & $(\mathrm{R} 4 \mathrm{a})$ \\
$\mathrm{S}_{\mathrm{L}}+\mathrm{TE} \rightleftharpoons 2 \mathrm{X}_{\mathrm{L}}$ & $(\mathrm{R} 4 \mathrm{~b})$ \\
$\mathrm{S}+\mathrm{X}_{\mathrm{D}} \rightleftharpoons \mathrm{S}_{\mathrm{D}}$ & $(\mathrm{R} 5 \mathrm{a})$ \\
$\mathrm{S}_{\mathrm{D}}+\mathrm{TE} \rightleftharpoons 2 \mathrm{X}_{\mathrm{D}}$ & $(\mathrm{R} 5 \mathrm{~b})$ \\
$\mathrm{X}_{\mathrm{L}}+\mathrm{X}_{\mathrm{D}} \rightleftharpoons \mathrm{P}+\mathrm{W}$ & $(\mathrm{R} 6)$ \\
$\mathrm{P} \rightleftharpoons 2 \mathrm{~S}$ & $(\mathrm{R} 7)$ \\
$\mathrm{W} \rightleftharpoons 2 \mathrm{~T}$ & $(\mathrm{R} 8)$ \\
\hline
\end{tabular}

The above model is a variation of the models in our earlier studies $[11,12,15]$ which are modifications of the original model of Frank [8]. The modifications allow us to analyze non-equilibrium symmetry breaking and rate of entropy production. Models such as this are used to extract general properties that are not model dependent. Examples of such properties are the qualitative behavior of steady-state rate of entropy production as a function of a parameter that drives the system away from equilibrium (such as the incident radiation intensity II in the above model). The difference in concentration between enantiomers of a chiral species as a function of a parameter, such as the intensity II, is generally parabolic, as predicted by bifurcation theory based on the symmetry group (mirror symmetry in this case) of the system, regardless of the details of the chemical reactions that break chiral symmetry.

Though there is currently no known reaction that has all the properties in the above model, the reaction has no steps that are implausible. For example, reactions (R1), (R1a), (R2) and (R3) comprise a photoaddition reaction that produces a chiral compound. An example is the following reaction series $[19,20]$ :

$$
\begin{gathered}
(\mathrm{Ph})_{2} \mathrm{C}=\mathrm{CH}\left(\mathrm{R}^{1}\right)+\mathrm{hv} \rightarrow\left[(\mathrm{Ph})_{2} \mathrm{C}=\mathrm{CH}\left(\mathrm{R}^{1}\right)\right]^{*} \\
{\left[(\mathrm{Ph})_{2} \mathrm{C}=\mathrm{CH}\left(\mathrm{R}^{1}\right)\right]^{*}+\left(\mathrm{R}^{2}\right) \mathrm{OH} \rightarrow\left[(\mathrm{Ph})_{2} \mathrm{HC}-\mathrm{CH}\left(\mathrm{R}^{1}\right)\left(\mathrm{OR}^{2}\right)\right]_{\mathrm{L}}} \\
{\left[(\mathrm{Ph})_{2} \mathrm{C}=\mathrm{CH}\left(\mathrm{R}^{1}\right)\right]^{*}+\left(\mathrm{R}^{2}\right) \mathrm{OH} \rightarrow\left[(\mathrm{Ph})_{2} \mathrm{HC}-\mathrm{CH}\left(\mathrm{R}^{1}\right)\left(\mathrm{OR}^{2}\right)\right]_{\mathrm{D}}}
\end{gathered}
$$

in which Ph is the phenyl group and $\mathrm{R}^{1}=\mathrm{CH}_{3}$ or $\mathrm{C}_{2} \mathrm{H}_{5}$ and $\mathrm{R}^{2}=\mathrm{CH}_{3}, \mathrm{C}_{2} \mathrm{H}_{5}$ or $\mathrm{C}_{3} \mathrm{H}_{7}$. In the reaction (R9), a photon is absorbed by the electrons in the $\mathrm{C}=\mathrm{C}$ double bond and the molecule transitions to a 
reactive excited state $\left[(\mathrm{Ph})_{2} \mathrm{C}=\mathrm{CH}\left(\mathrm{R}^{1}\right)\right]^{*}$. In the addition reaction shown in (R10) and (R11), the excited molecule reacts with an alcohol, $\left(\mathrm{R}^{2}\right) \mathrm{OH}$, and produces a chiral compound $(\mathrm{Ph})_{2} \mathrm{HC}-\mathrm{CH}\left(\mathrm{R}^{1}\right)\left(\mathrm{OR}^{2}\right)$ in the $\mathrm{L}$ and $\mathrm{D}$ enantiomeric forms. In this compound, the carbon shown in boldface is a chiral carbon (its tetrahedral bonds to four different groups makes it so). Other examples of photoaddition reactions producing chiral products from achiral reactants can be found in [19]. We note that TE need not be an excited state; it could be a different, more reactive isomer of the T [19].

The reactions (R4a)-(R5b) are steps leading to chiral autocatalysis. This involves the formation of a chiral complex of $S$ and $X$ in their enantiomeric forms. Examples of chiral complexes resulting in reactions with a high degree of chiral selectivity have been known for a long time [21,22]. In an article published in 1984, we noted some mechanisms that are based on chiral ligands in rhodium phosphine catalysts which could lead to chiral autocatalysis [12,22]. To date, there are several chirally autocatalytic reactions that have been experimentally studied. Chiral symmetry breaking was noticed and systematically studied first in $\mathrm{NaClO} 3$ in 1990 [23], and in 1995 chiral autocatalysis and amplification of small initial enantiomeric excess was reported in inorganic reactions involving cobalt complexes [24] and in organic reactions involving alkylation of aldehydes [25]. Since then, these and closely related systems have been extensively experimentally studied and the mechanisms of chiral autocatalysis have been investigated [26-31]. A variant of chiral symmetry breaking in stirred crystallization was reported in 2005, and it too has been studied extensively [32,33]. The mechanisms of chiral autocatalysis vary in these systems: in crystallization, it is secondary nucleation, in the organic and inorganic reactions, cluster/complex formation seems to be involved. Reactions (R4a) and (R5a) may be thought of as a simple form of chiral complex formation. As was noted in a review [27], the exact details of chiral catalysis are not of significance for the general properties symmetry-breaking bifurcation and thermodynamic properties of such systems. In particular, properties such as phase-transitions-like behavior we present here are quite independent of the details of chemical kinetics. Examples of reaction (R6), the dimer formation of enantiomers, are also known; in fact, such dimerization of certain chiral catalysts leads to asymmetric amplification [34].

For the above theoretical model (R1)-(R8), the corresponding forward and reverse rate for each reaction are written as follows, in which concentrations are shown explicitly as functions of time:

$$
\begin{aligned}
& \mathrm{R} 1 \mathrm{f}=(\mathrm{k} 1 \mathrm{f}+\mathrm{k} 1 \mathrm{II}) \mathrm{T}[\mathrm{t}], \mathrm{R} 1 \mathrm{r}=\mathrm{k} 1 \mathrm{r} \mathrm{TE}[\mathrm{t}] \\
& \mathrm{R} 2 \mathrm{f}=\mathrm{k} 2 \mathrm{f} \mathrm{S}[\mathrm{t}] \mathrm{TE}[\mathrm{t}], \mathrm{R} 2 \mathrm{r}=\mathrm{k} 2 \mathrm{r} X_{\mathrm{L}}[\mathrm{t}] \\
& \mathrm{R} 3 \mathrm{f}=\mathrm{k} 3 \mathrm{f} \mathrm{S}[\mathrm{t}] \mathrm{TE}[\mathrm{t}], \mathrm{R} 3 \mathrm{r}=\mathrm{k} 3 \mathrm{r} \mathrm{X}_{\mathrm{D}}[\mathrm{t}] \\
& \mathrm{R} 4 \mathrm{af}=\mathrm{k} 4 \mathrm{af} \mathrm{S}[\mathrm{t}] \mathrm{X}_{\mathrm{L}}[\mathrm{t}], \mathrm{R} 4 \mathrm{ar}=\mathrm{k} 4 \mathrm{ar} \mathrm{S}_{\mathrm{L}}[\mathrm{t}] \\
& \mathrm{R} 4 \mathrm{bf}=\mathrm{k} 4 \mathrm{bf} \mathrm{S}_{\mathrm{L}}[\mathrm{t}] \mathrm{TE}[\mathrm{t}], \mathrm{R} 4 \mathrm{br}=\mathrm{k} 4 \mathrm{br}\left(\mathrm{X}_{\mathrm{L}}[\mathrm{t}]\right)^{2} \\
& \mathrm{R} 5 \mathrm{af}=\mathrm{k} 5 \mathrm{af} S[\mathrm{t}] \mathrm{X}_{\mathrm{D}}[\mathrm{t}], \mathrm{R} 5 \mathrm{ar}=\mathrm{k} 5 \mathrm{ar} \mathrm{S}_{\mathrm{D}}[\mathrm{t}] \\
& \mathrm{R} 5 \mathrm{bf}=\mathrm{k} 5 \mathrm{bf} \mathrm{S}_{\mathrm{D}}[\mathrm{t}] \mathrm{TE}[\mathrm{t}], \mathrm{R} 5 \mathrm{br}=\mathrm{k} 5 \mathrm{br}\left(\mathrm{X}_{\mathrm{L}}[\mathrm{t}]\right)^{2} \\
& R 6 f=k 6 f X_{L}[t] X_{D}[t], R 6 r=k 6 r P[t] W[t] \\
& \mathrm{R} 7 \mathrm{f}=\mathrm{k} 7 \mathrm{f} \mathrm{P}[\mathrm{t}], \mathrm{R} 7 \mathrm{r}=\mathrm{k} 7 \mathrm{r}(\mathrm{S}[\mathrm{t}])^{2} \\
& \mathrm{R} 8 \mathrm{f}=\mathrm{k} 8 \mathrm{f} \mathrm{W}[\mathrm{t}], \mathrm{R} 8 \mathrm{r}=\mathrm{k} 8 \mathrm{r}(\mathrm{T}[\mathrm{t}])^{2}
\end{aligned}
$$

In these equations, the rate constants are written as $k 1 \mathrm{f}, \mathrm{k} 1 \mathrm{r}$ etc., and the concentration are written as $\mathrm{T}[\mathrm{t}], \mathrm{S}[\mathrm{t}]$, etc. In terms of these forward and reverse rates, the rate equations for the concentrations can be written as:

$$
\begin{gathered}
d T[t] / d t=-R 1 f+R 1 r+2 R 8 f-2 R 8 r \\
d T E[t] / d t=-R 1 f+R 1 r-R 2 f+R 2 r-R 3 f+R 3 r-R 4 b f+R 4 b r-R 5 b f+R 5 b r
\end{gathered}
$$




$$
\begin{gathered}
d S[t] / d t=-R 2 f+R 2 r-R 3 f+R 3 r-R 4 a f+R 4 a r-R 5 a f+R 5 a r+2 R 7 f-2 R 7 r \\
d S_{L}[t] / d t=R 4 a f-R 4 a r-R 4 b f+R 4 b r \\
d S_{D}[t] / d t=R 5 a f-R 5 a r-R 5 b f+R 5 b r \\
d X_{L}[t] / d t=R 2 f-R 2 r-R 4 a f+R 4 a r+2 R 4 b f-2 R 4 b r-R 6 f+ \\
d X_{D}[t] / d t=R 3 f-R 3 r-R 5 a f+R 5 a r+2 R 5 b f-2 R 5 b r-R 6 f+R 6 r \\
d P[t] / d t=R 6 f-R 6 r-R 7 f+R 7 r \\
d W[t] / d t=R 6 f-R 6 r-R 8 f+R 8 r
\end{gathered}
$$

This set of coupled non-linear equations were solved numerically using Mathematica NDSolve. NDSolve is a Mathematica command that has the following structure: NDSolve[\{Equations $\},\left\{y_{i}\right\},\{t$, $\left.\left.t_{\min }, t_{\max }\right\}\right]$, in which "Equations" are the differentials equations for the set of functions $\left\{\mathrm{y}_{\mathrm{i}}\right\}$ with $\mathrm{t}$ as the independent variable; numerical solutions are obtained in the range $t_{\min }$, to $t_{\max }$. More details can be found in the online documentation that comes with Mathematica. The rate constants used for the numerical solutions are summarized in Figure 1. In assigning values to rate constants, there are consistency conditions that must be met. For example, since reactions (R4a) and (R4b) together are equivalent to (R2), the products of the equilibrium constants of R4a and R4b must equal the equilibrium constant of R2. This gives us the following condition for the rate constants:

$$
(\mathrm{k} 4 \mathrm{af} / \mathrm{k} 4 \mathrm{ar})(\mathrm{k} 4 \mathrm{bf} / \mathrm{k} 4 \mathrm{br})=\mathrm{k} 2 \mathrm{f} / \mathrm{k} 2 \mathrm{r}
$$

$$
\begin{array}{ll}
k 1 f=\left(10^{-3}\right) k 1 r & k 5 a f=k 4 a f \\
k 1 r=1.0 & k 5 a r=k 4 a r \\
k I=1 & k 5 b f=k 4 b f \\
k 2 f=0.5 & k 5 b r=k 4 b r \\
k 2 r=0.001 & k 6 r=k 6 f\left(\frac{k 1 f}{k 1 r}\right)^{2}\left(\frac{k 2 f}{k 2 r}\right)\left(\frac{k 3 f}{k 3 r}\right)\left(\frac{k 7 f}{k 7 r}\right)\left(\frac{k 8 f}{k 8 r}\right) \\
k 3 f=k 2 f & k 7 f=0.5 \\
k 3 r=k 2 r & k 7 r=5.0 \\
k 4 a f=10^{6} & k 8 f=k 7 f \\
k 4 a r=10^{5} & k 8 r=k 7 r \\
k 4 b f=10^{3} & I I=\text { variable } \\
k 4 b r=k 4 b f\left(\frac{k 4 a f}{k 4 a r}\right)\left(\frac{k 2 r}{k 2 f}\right) & k 90.0
\end{array}
$$

Figure 1. The figure shows numerical values assigned to rate constants to obtain numerical solution for the rate equations of the system. Units of volume are assumed to be liters (L) and concentrations mol/L (M). Units of variable parameter II may be thought of as $\mathrm{W} / \mathrm{m}^{2}$. If II is thought of as a radiation from the sun, its blackbody temperature is very high compared to the temperature of the system. All rate constants are assumed to have the appropriate units, though not written explicitly. 
Numerical values were assigned to rate constants so as to fulfill these requirements. The units were chosen so that all concentrations are in $\mathrm{mol} / \mathrm{L}$. Assigned numerical values are such that the concentrations of the reactants have realistic values. Symmetric (racemic) and asymmetric states are parametrized by $\alpha=\left(X_{L}-X_{D}\right)$, in the symmetric state $\alpha=0$ and in the asymmetric state $\alpha \neq 0$.

\section{Results}

The rate equations were first solved for an equilibrium state where the incident radiation intensity, II, was set to 0 . The initial concentrations of species $\mathrm{S}$ and $\mathrm{T}$ were set to $0.01 \mathrm{M}$ and the initial concentrations of all other species were set to $0.0 \mathrm{M}$. Under these conditions, the system evolves to its racemic equilibrium state, in which $\alpha=0$.

The simulation code was run for sufficient time (about $10^{4} \mathrm{~s}$ ) to ensure the concentrations of all species have reached a steady state, which is the equilibrium state. At $\mathrm{t}=10^{4} \mathrm{~s}$, the concentrations at equilibrium were: $\mathrm{S}=\mathrm{T}=8.478 \times 10^{-3} \mathrm{M}, \mathrm{TE}=8.477 \times 10^{-6} \mathrm{M}, \mathrm{S}_{\mathrm{L}}=\mathrm{S}_{\mathrm{D}}=3.047 \times 10^{-6} \mathrm{M}$, $\mathrm{X}_{\mathrm{L}}=\mathrm{X}_{\mathrm{D}}=3.593 \times 10^{-5} \mathrm{M}$, and $\mathrm{P}=\mathrm{W}=7.188 \times 10^{-4} \mathrm{M}$. The conversion of the initial species $\mathrm{S}$ and $\mathrm{T}$ compared to other species was rather small for the numerical values of the rate constants shown in Figure 1. By choosing a different set of rate constants, the conversion could be increased. The numerical values confirm that complete symmetry of the system was maintained when no incident radiation is present. Figure 2 shows the time evolution of the chiral species $S_{L}, S_{D}, X_{L}, X_{D}$ from $t=0 s$, to $t=1000 s$. As the system evolved to its equilibrium state, the entropy production $\sigma$ was monitored; initially, it took a nonzero value but, as expected, its value decreased to zero at the equilibrium state.

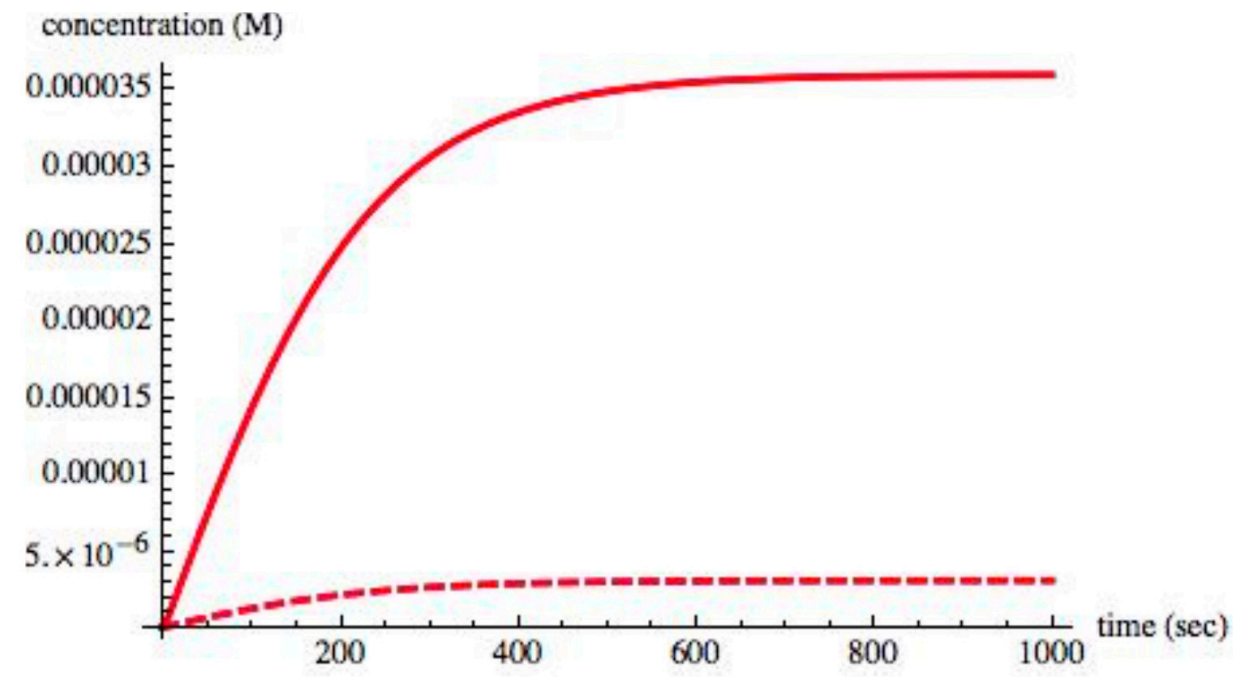

Figure 2. Time evolution of chiral species to their equilibrium state in the model reaction scheme when $\mathrm{II}=0$ and $\mathrm{S}=\mathrm{T}=0.01 \mathrm{M}$ at $\mathrm{t}=0$. Concentrations of all other species was set to zero at $\mathrm{t}=0$. Due to the symmetry of the system, at equilibrium, $S_{L}=S_{D}$ (dashed line) and $X_{L}=X_{D}$ (solid line), so the curves for the two enantiomers overlap.

We then used these values for a symmetric equilibrium state as initial values for a system subject to a radiation input, i.e., II $>0$. This radiation input serves as a means to push the system away from thermodynamic equilibrium. A very small excess, about $0.1 \%\left(3 \times 10^{-8} \mathrm{M}\right)$ of $\mathrm{X}_{\mathrm{L}}$ was introduced into the system as a "random fluctuation". If the system has the mechanism to break chiral symmetry, it will have a critical value $\mathrm{II}_{\mathrm{C}}$. At values of $\mathrm{II}<\mathrm{II}_{\mathrm{C}}$, this excess $0.1 \%$ of $\mathrm{X}_{\mathrm{L}}$ will decrease and the system will again evolve into a steady state where $X_{L}=X_{D}$; at values of II $>I_{C}$, the excess will increase and lead to a steady state in which $X_{L}>X_{D}$. In a real system, this slight perturbation may be due to a random fluctuation such as a local excess of one enantiomer that may then be amplified, resulting in a state of broken symmetry. The overall behavior of the system is summarized in Figure 3. 


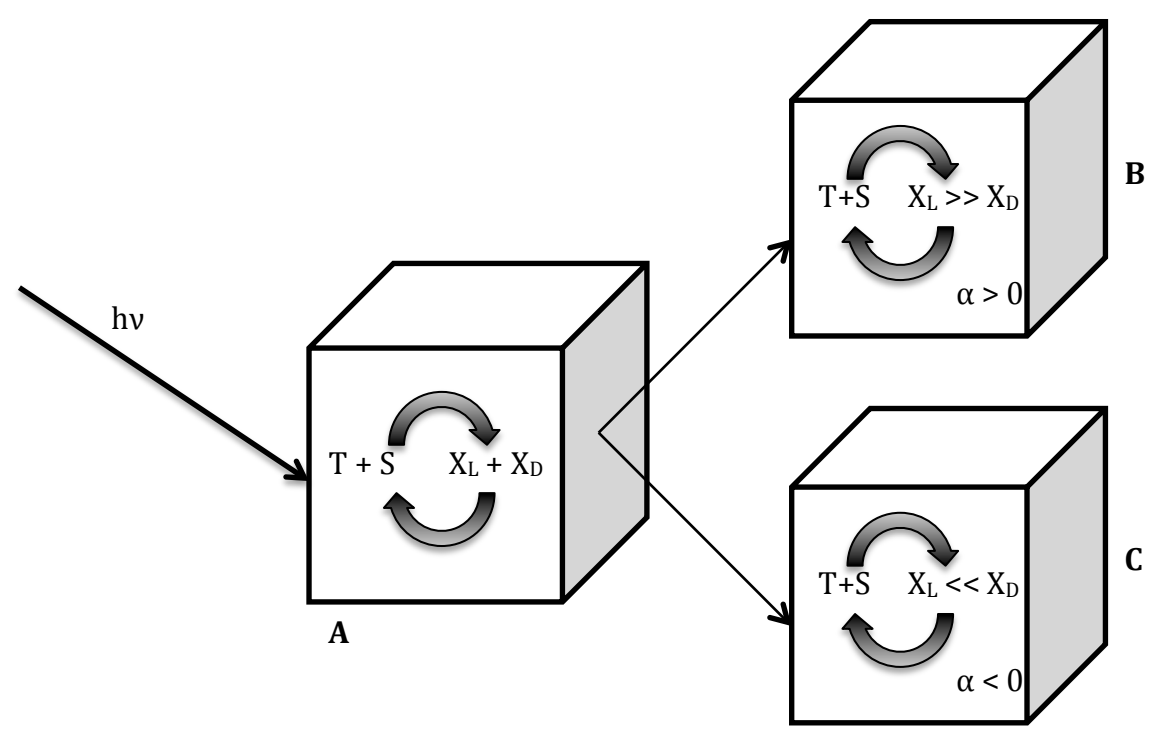

Figure 3. Schematic of reaction system. Radiation (shown as hv and denoted as II in the reaction scheme)) is incident on a closed chemical system. The radiation drives a generation-decomposition cycle of enantiomeric species $X$ and other compounds, as shown in $\mathrm{A}$. When $\mathrm{II}>\mathrm{II}_{\mathrm{C}}$, the system evolves to one of two asymmetric states, $B$ or $C$. In state $B$, the amount of $X_{L}>X_{D}$, and in state $C, X_{D}>X_{L}$. The asymmetry is parametrized by $\alpha=\left(X_{L}-X_{D}\right)$.

It was found that this system indeed has the mechanism needed for breaking chiral symmetry. At values of II $<0.004$, the system evolved to a symmetric state corresponding to $\mathrm{X}_{\mathrm{L}}=\mathrm{X}_{\mathrm{D}}$ and $\alpha=0$. Figure 4 shows the time evolution of the chiral species when II $=0.0030$. A steady state is reached in approximately $600 \mathrm{~s}$.

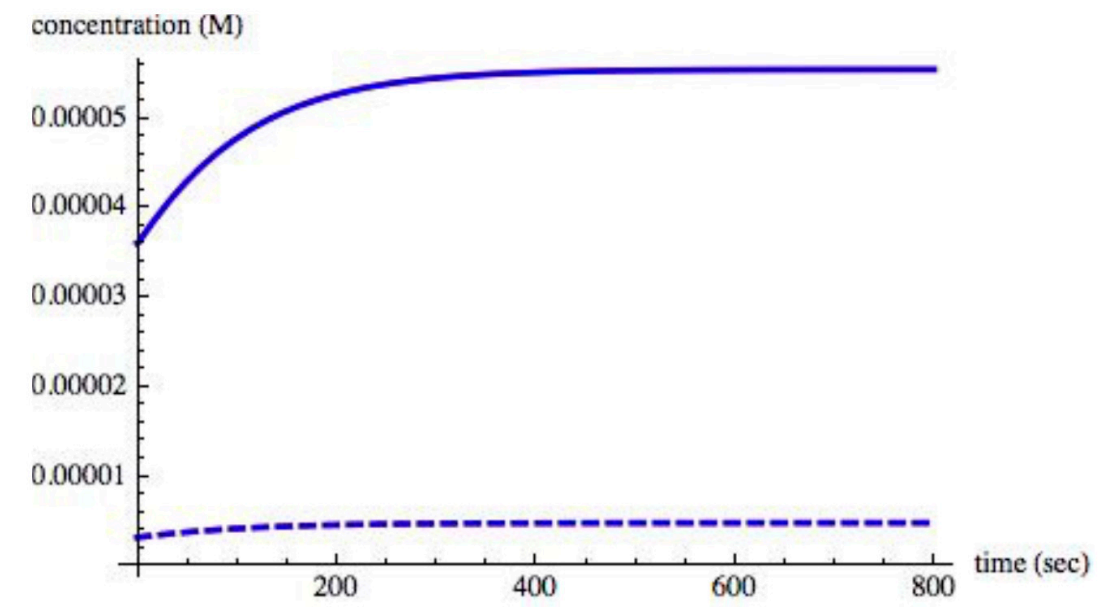

Figure 4. Time evolution of chiral species using equilibrium values for initial conditions when II $=0.003$. The solid line represents $X_{L}$ and $X_{D}$ and the dashed $S_{L}$ and $S_{D}$. Since symmetry is not broken, the amounts of each chiral species are exactly equal, hence the overlapping curves $(\alpha=0)$.

For values of II $>0.004$, the small excess of $0.1 \%$ of $\mathrm{XL}$ in the initial concentration increased, thus driving all chiral species to an asymmetric state. A time evolution of the chiral species in an asymmetric state when II $=0.008$ is shown in Figure 5. 


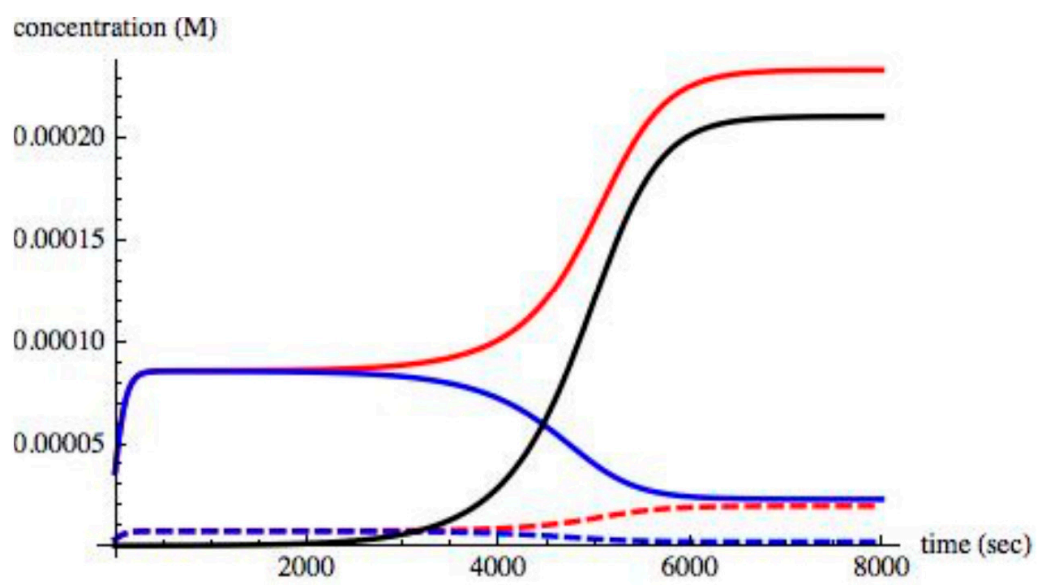

Figure 5. Time evolution of chiral species in an asymmetric state. Here, $\mathrm{II}=0.008$ and an asymmetric steady state is reached in about $7000 \mathrm{~s}$. The blue solid and dashed lines represent $X_{D}$ and $S_{D}$, respectively, and the red solid and dashed lines represent $X_{L}$ and $S_{L}$, respectively. The black line represents $\alpha$, which takes on a nonzero value once symmetry is broken.

As shown, steady state is reached about $7000 \mathrm{~s}$. The time taken to reach steady state, the relaxation time, depends on the value of II and on the amount of initial excess $\left(0.1 \%\right.$ of $\left.\mathrm{X}_{\mathrm{L}}\right)$. As is well known in the study of stability of steady states $[6,7,9]$, the initial exponential growth of the small enantiomeric excess depends on the eigenvalue of the unstable mode of the linearized equations derived from the set (14)-(22) around the initial state. These linearized equations are obtained by assuming a small perturbation of the concentrations, $\delta C_{k}$, from the initial steady state. This leads to a set of linear equations of the type $d \delta C_{k} / d t=\Sigma_{1} L_{k l} \delta C_{1}[6,7,9]$. The eigenvalues of $L_{k l}$ with positive real parts are the exponents that determine the initial rate of growth of the enantiomeric excess. However, the later growth and leveling off at the steady state depend on the kinetics and rate constants. In general, near the critical point $\mathrm{II}_{\mathrm{C}}$, the relaxation time is long, the so-called "critical slowing", but as the value of II increases, the growth rate becomes faster and the relaxation time decreases.

To determine the relation between the steady-state value of $\alpha$ on II, the reaction was run for various values of II, from 0.0035 to 0.0045 , and the corresponding steady-state values of $\alpha$ were obtained. As noted above, the time it takes for the system to reach an asymmetric steady state depends on the value of II; close to the critical value $\mathrm{II}_{\mathrm{C}}(0.004$ in this case), the relaxation to asymmetric steady state is slower and it becomes faster as the value of II increases. The exact quantitative relationship between relaxation time and II depends on the kinetics and rate constants and not of significance to the current study. In these runs, to obtain both positive and negative branches of $\alpha$, the initial condition with a $0.1 \%$ excess of $X_{D}$ was also included. The dependence of $\alpha$ on II is shown in Figure 6, demonstrating the typical bifurcation of asymmetric states above the critical value $\mathrm{II}_{C}=0.004$. As is expected, in a chiral symmetry breaking transition, the values of $\alpha$ above the critical point are parabolic.

With these results, we now turn to the rate of entropy production $\sigma$. As stated above, initially the system is in the state of equilibrium, with the intensity of radiation II $=0$ and $\sigma=0$. Then the intensity of the radiation II is increased to a non-zero value and the rate of entropy production $\sigma$ is monitored. Initially, $\sigma$ sharply increases and, as the system reaches its steady-state, corresponding to the set value of II, the entropy production also reaches its steady state value. Figure 7 shows the evolution of $\sigma$ from its value when $t=0$, to its final steady-state value when $I I=0.008$. The final steady state value of $\sigma$ is small compared to its initial value, but it is nonzero. 


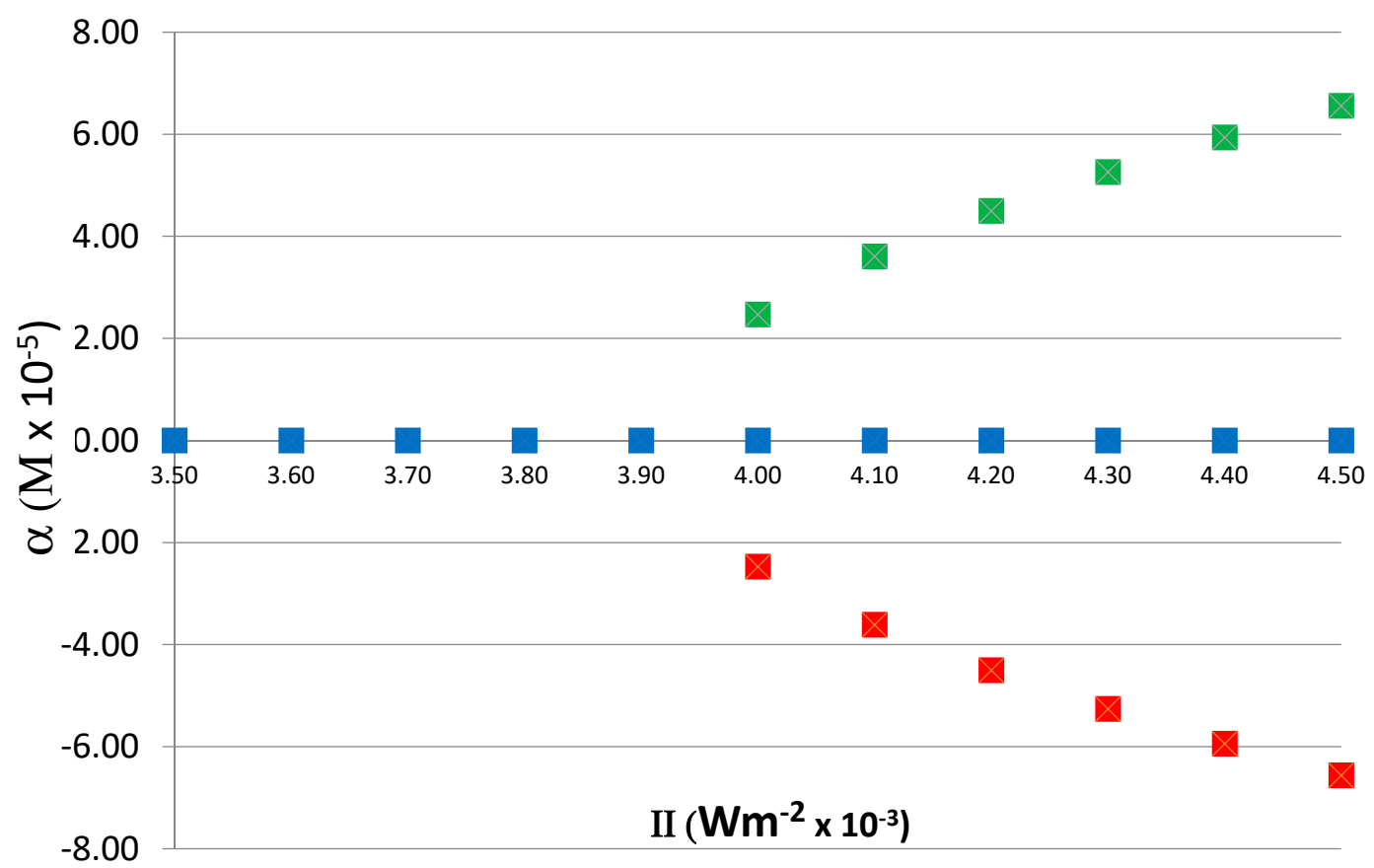

Figure 6. Dependence of $\alpha$ on II. Units of $\alpha$ are $\mathrm{M}$ and II are $\mathrm{Wm}^{-2}$. Steady-state values of $\alpha$ are plotted as a function of II. When II $>\mathrm{II}_{\mathrm{C}}, \alpha$ takes a positive $\left(\mathrm{X}_{\mathrm{L}}>\mathrm{X}_{\mathrm{D}}\right.$, shown in green $\left.\mathrm{X}\right)$ or negative $\left(\mathrm{X}_{\mathrm{L}}<\mathrm{X}_{\mathrm{D}}\right.$, shown in red $\mathrm{X}$ ) value, depending on the random perturbation that drives the system away from the unstable racemic state $\alpha=0$. The blue Xs show the symmetric branch which is unstable above the critical point. In the region $\mathrm{II}>\mathrm{II}_{\mathrm{C}}, \alpha$ increases in a characteristically parabolic manner.

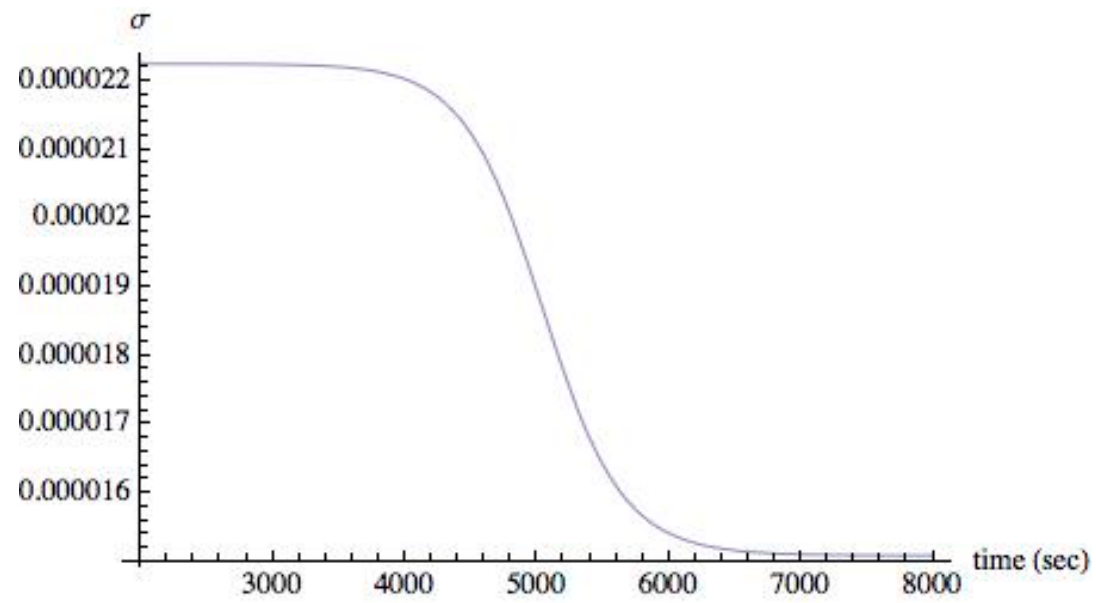

Figure 7. Rate of entropy production in an asymmetric state when $\mathrm{II}=0.008$. Units of $\sigma$ are $\mathrm{JK}^{-1} \mathrm{~L}^{-1} \mathrm{~s}^{-1}$. As in Figure 3, steady state is reached at about $7000 \mathrm{~s}$. Although it may appear that $\sigma$ is approaching 0 , it is not so; $\sigma$ maintains a nonzero value at steady state after symmetry is broken.

We would like to note that the results shown above do not depend crucially on the particular values assigned to rate constants. Whether symmetry breaking occurs or does not depends mostly on the mechanism of reactions in the model, not on a narrow range of values of rate constants. In general, qualitative properties change drastically due to small changes in rate constants only in singular cases. The presented model is not singular. In our study, we have tried a range of rate constants and observed symmetry breaking. A typical value is presented in this article.

Our objective is to study the behavior of $\sigma$ as the intensity of radiation II moves form a value below to a value above the critical value $\mathrm{II}_{\mathrm{C}}$. In a previous study [18] of an open system, $\sigma$ behaved as entropy does in a second order phase transition: its slope is discontinuous at the transition point. 
In addition, we noted that its slope increased above the critical point. This behavior was consistent with the Maximum Entropy Production (MEP) hypothesis [35-40] that states that non-equilibrium steady states maximize the rate of entropy production. In other words, the breaking of symmetry was a reflection of the general tendency of non-equilibrium systems and, from this point of view, it was only to be expected. This would imply that biochemical asymmetry is a consequence of MEP. To date, there is no general proof for MEP; indeed, in our own investigation we found that MEP was valid for some systems but not all. MEP, in general, is a controversial hypothesis and its applications to various complex systems have been questioned [41]. Hence, we investigated MEP in the context of chiral symmetry breaking.

Figure 8 shows the behavior of entropy production in the closed system we present here. It shows a change in the slope at the transition point, IIC, as in our previous study. From the point of view of symmetry breaking transition, we see that entropy production behaves in a way that is similar to that of entropy in a second-order phase transition. However, unlike the previous result, the slope decreases above the critical point. As indicated in Figure 8, when II $>\mathrm{II}_{\mathrm{C}}$, if initially we set the system in a non-equilibrium symmetric state, which is an extrapolation of the symmetric state below $\mathrm{II}_{\mathrm{C}}$, the system evolves to an asymmetric state in which $\sigma$ is lower. The extrapolation of the symmetric state beyond the critical value $\mathrm{II}_{C}$ is possible on a computer because, without a small perturbation in $X_{L}$ or $X_{D}$, or other chiral species, the system stays in an unstable symmetric steady state. With a small perturbation, it evolves to the stable asymmetric state. The time evolution of the system from this initial state to its final asymmetric steady state results in the decrease in $\sigma$, thus indicating that the stable asymmetric state is associated with a lower value of $\sigma$ compared with that of a symmetric state. Thus, we see that the entropy production in a closed system is not consistent with MEP, because MEP would predict higher values for $\sigma$ in the asymmetric state.

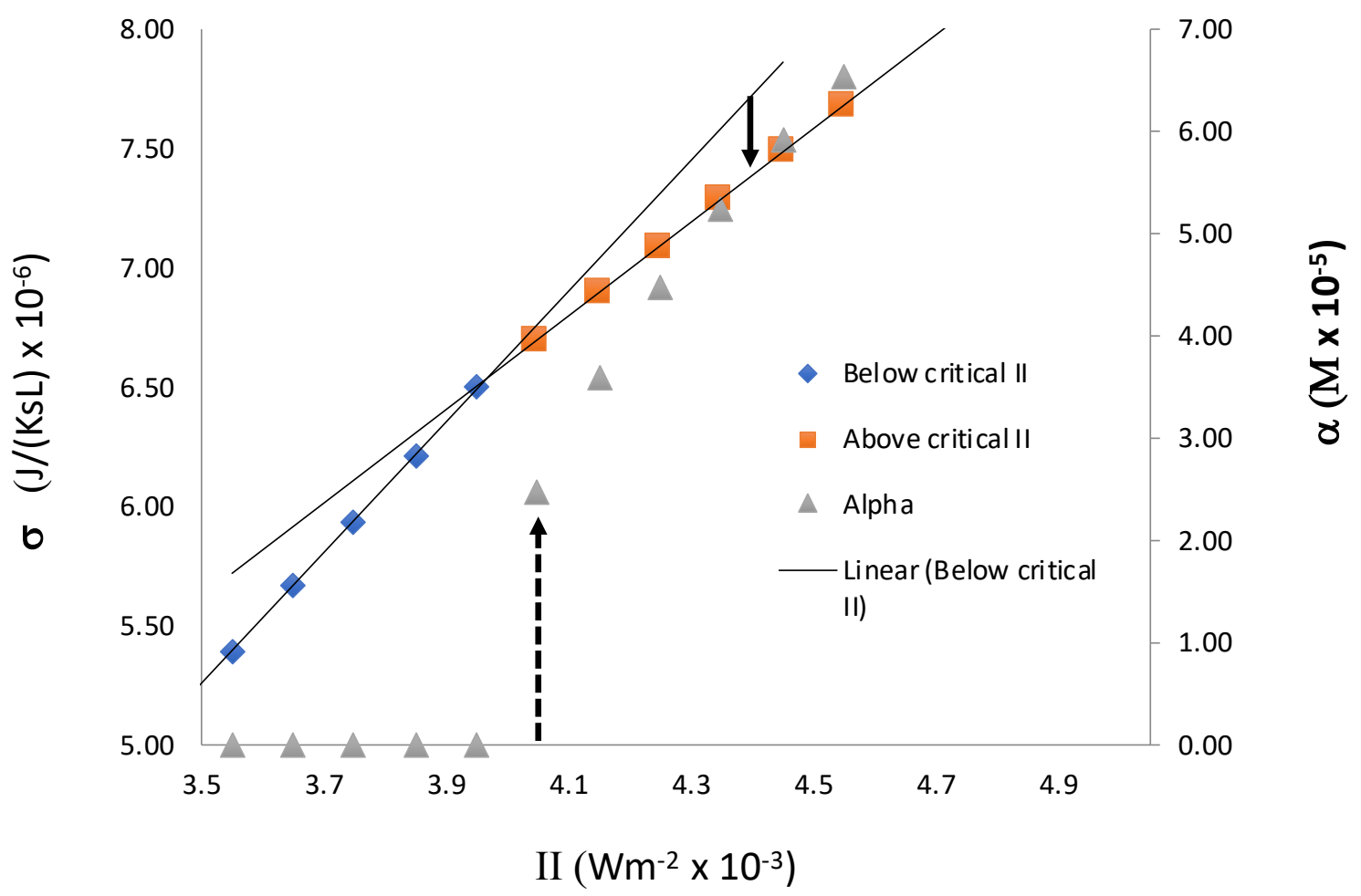

Figure 8. Rate of entropy production $\sigma$ and $\alpha$ as a function of II. The critical value $\mathrm{II}_{\mathrm{C}}=0.0040$. Beyond this point, $\alpha$ takes on nonzero values and there is a decrease in the slope of $\sigma$. Units of II are $\mathrm{Wm}^{-2}$. The dashed arrow indicates a transition from an unstable state, where $\alpha=0$, to a stable asymmetric state where $\alpha$ is nonzero. In the region $\mathrm{II}>\mathrm{II}_{\mathrm{C}}$ rate of entropy production of asymmetric state is lower than that of the symmetric state. The solid arrow shows that transition from an unstable symmetric state to a stable asymmetric state results in the lowering of $\sigma$, the rate of entropy production. 


\section{Concluding Remarks}

Our results show several aspects of chiral symmetry breaking. First, they show that Frank's original model can be modified with several additional steps-all of which are possible elementary chemical reaction steps-to demonstrate that spontaneous chiral symmetry breaking can occur in a photochemically driven closed system. Our model is motivated by the fact that the earth is a essentially a closed system (except for a very small influx in interstellar matter such as meteorites) and the evolution of life was driven by incident solar radiation. The incident radiation drives a cycle of generation and decomposition of chiral compounds that, at a sufficiently high intensity of radiation, makes a transition to a state of broken chiral symmetry. This example demonstrates that life on earth could have evolved under such conditions of prebiotic molecular chiral asymmetry.

From a thermodynamic viewpoint, this study also confirms that chiral symmetry breaking transitions are similar to second-order phase transitions, with entropy production taking the place of entropy. We note that the general qualitative features of bifurcation of asymmetric states and change in the behavior entropy production at the critical point, shown in Figures 6 and 8, are a consequence of the two-fold symmetry of the system, not the particularities of the chemical reactions in the model. In fact, using the system's symmetry group, it is possible to derive the following generic equation for the evolution of $\alpha$ near the critical point: $\mathrm{d} \alpha / \mathrm{dt}=-\mathrm{A} \alpha^{3}+\mathrm{B} \alpha+\mathrm{C}$, in which $\mathrm{A}, \mathrm{B}$ and $\mathrm{C}$ are functions of the kinetic constants of the chiral symmetry breaking chemical reactions [11-13,15]. Finally, we show that the behavior of entropy production in this chiral-symmetry-breaking system is not consistent with the MEP hypothesis, because MEP implies that the state of broken symmetry will have a higher rate of entropy production compared to a symmetric state, but we find the opposite to be the case in this model. In a system with an inflow of reactants and outflow of products, however, the entropy production was higher in the asymmetric state. This indicates that MEP is valid for a certain class of systems, but what this class is has not yet been clearly identified.

Author Contributions: Conceptualization, D.K.; methodology, D.K. and Z.M.; software and Mathematica Code, D.K. \& Z.M. All authors have read and agreed to the published version of the manuscript.

Funding: Wake Forest University, Winston Salem, NC 27109, USA.

Conflicts of Interest: The authors declare no conflict of interest.

\section{References}

1. Onsager, L. Reciprocal Relations in Irreversible Processes. Phys. Rev. 1931, 37, 405-426. [CrossRef]

2. De Donder, T.; Van Rysselberghe, P. Affinity; Stanford University Press: Menlo Park, CA, USA, 1936.

3. Prigogine, I. Etude Thermodynamique des Phénomènes Irréversibles; Editions Desoer: Liège, Belgium, 1947.

4. Prigogine, I. Introduction to Thermodynamics of Irreversible Processes; John Wiley: London, UK, 1967.

5. De Groot, S.R.; Mazur, P. Non-Equilibrium Thermodynamics; North Holland Press: Amsterdam, The Netherlands, 1969.

6. Kondepudi, D.K.; Prigogine, I. Modern Thermodynamics: From Heat Engines to Dissipative Structures, 2nd ed.; John Wiley: New York, NY, USA, 2015.

7. Nicolis, G.; Prigogine, I. Self-Organization in Non-equilibrium Systems; Wiley: New York, NY, USA, 1977.

8. Frank, F.C. Spontaneous asymmetric synthesis. Biochem. Biophys. Acta 1953, 11, 459-463. [CrossRef]

9. Haken, H. Synergetics: An Introduction; Springer: Berlin, Germany, 1977.

10. Kondepudi, D.K.; Hegstrom, R.A. The Handedness of the Universe. Sci. Am. 1990, 262, 108-115.

11. Kondepudi, D.K.; Nelson, G.W. Chiral Symmetry Breaking in Nonequilibrium Systems. Phys. Rev. Lett. 1983, 50, 1023-1026. [CrossRef]

12. Kondepudi, D.K.; Nelson, G. Chiral Symmetry Breaking states and Their Sensitivity in Nonequilibrium Systems. Physica A 1984, 125, 465-496. [CrossRef]

13. Kondepudi, D.K. State Selection Dynamics in Symmetry Breaking Transitions. In Noise in Nonlinear Dynamical Systems; Moss, F., McClintock, P.V., Eds.; Cambridge University Press: Cambridge, UK, 1989; Volume 2, pp. 251-270. 
14. Hegstrom, R.A.; Rein, D.W.; Sanders, P.G.H. Calculation of the parity nonconserving energy difference between mirror-image molecules. J. Chem. Phys. 1980, 73, 2329-2341. [CrossRef]

15. Kondepudi, D.K.; Nelson, G.W. Weak Neutral Currents and the Origin of Biomolecular Chirality. Nature 1985, 314, 438-441. [CrossRef]

16. Decker, $\mathrm{P}$. The origin of molecular asymmetry through the amplification of "stochastic information" (noise) in bioids, open systems which can exist in several steady states. J. Mol. Evol. 1974, 4, 49-65. [CrossRef]

17. Plasson, R.; Kondepudi, D.K.; Bersini, H.; Commeyras, A.; Asakura, K. Emergence of homochirality in far-for-equilibrium systems: Mechanisms and role in prebiotic chemistry. Chirality 2007, 19, 589-600. [CrossRef]

18. Kondepudi, D.K.; Kapcha, L. Entropy production in chiral symmetry breaking transitions. Chirality 2008, 20, 524-528. [CrossRef]

19. Inoue, Y. Enantiodifferentiating Photosensitized Reactions. In Chiral Photochemistry; CRC Press: Boca Raton, FL, USA, 2004; Chapter 4.

20. Inoue, Y.; Ramamurthy, V. (Eds.) Chiral Photochemistry; Marcel Decker: New York, NY, USA, 2004.

21. Kagan, H.B. Chiral Ligands for Asymmetric Catalysis. In Asymmetric Synthesis: Chiral Catalysis; Morrison, J.D., Ed.; Academic Press: New York, NY, USA, 1985; Chapter 1; Volume 5, pp. 1-39.

22. Fryzuk, M.D.; Bosnich, B. Asymmetric synthesis. An asymmetric homogeneous hydrogenation catalyst which breeds its own chirality. J. Am. Chem. Soc. 1978, 100, 5491-5494. [CrossRef]

23. Kondepudi, D.K.; Kaufmann, R.; Singh, N. Chiral Symmetry Breaking in Sodium Chlorate Crystallization. Science 1990, 250, 975-976. [CrossRef] [PubMed]

24. Asakura, K.; Kobayashi, K.; Mizusawa, Y.; Ozawa, T.; Osanai, S.; Yoshikawa, S. Generation of an optically active octahedral cobalt complex by a chiral autocatalysis. Physica D 1995, 84, 72-78. [CrossRef]

25. Soai, K.; Shibata, T.; Morioka, H.; Choji, K. Asymmetric autocatalysis and amplification of enantiomeric excess of a chiral molecule. Nature 1995, 378, 767-768. [CrossRef]

26. Kondepudi, D.K.; Asakura, K.; Martin, R. Mechanism of Chiral Asymmetry Generation by Chiral Autocatalysis in the Preparation of Chiral Octahedral Cobalt Complex. Chirality 1998, 10, 343-348.

27. Kondepudi, D.K.; Asakura, K. Chiral Autocatalysis, Spontaneous Symmetry Breaking, and Stochastic Behavior. Acc. Chem. Res. 2001, 34, 946-954. [CrossRef]

28. Soai, K.; Shibata, T.; Sato, I. Enantioselective Automultiplication of Chiral Molecules by Asymmetric Autocatalysis. Acc. Chem. Res. 2000, 33, 382-390. [CrossRef]

29. Gehring, T.; Busch, M.; Schlageter, M.; Weingand, D. A concise summary of experimental facts about the Soai reaction. Chirality 2010, 22 (Suppl. 1), E173-E182. [CrossRef]

30. Soai, K.; Kawasaki, T.; Matsumoto, A. Asymmetric Autocatalysis of Pyrimidyl Alkanol and Its Application to the Study on the Origin of Homochirality. Acc. Chem. Res. 2014, 47, 3643-3654. [CrossRef]

31. Blackmond, D.G. Autocatalytic Models for the Origin of Biological Homochirality. Chem. Rev. 2019. [CrossRef]

32. Viedma, C. Chiral Symmetry Breaking During Crystallization: Complete Chiral Purity Induced by Nonlinear Autocatalysis and Recycling. Phys. Rev. Lett. 2005, 94, 065504-065506. [CrossRef] [PubMed]

33. Sögütoglu, L.C.; Steendam, R.R.; Meekes, H.; Vlieg, E.; Rutjes, F.P. Viedma ripening: A reliable crystallisation method to reach single chirality. Chem. Soc. Rev. 2015, 44, 6723-6732. [CrossRef] [PubMed]

34. Girard, C.; Kagan, H. Nonlinear Effects in Asymmetric Synthesis and Stereoselective Reactions: Ten Years of Investigation. Angew. Chem. Int. Ed. 1998, 37, 2922-2959. [CrossRef]

35. Swenson, R. Emergent attractors and the law of maximum entropy production: Foundations to a theory of general evolution. Syst. Res. 1989, 6, 187-197. [CrossRef]

36. Swenson, R.; Turvey, M.T. Thermodynamic Reasons for Perception-Action Cycles. Ecol. Psychol. 1991, 3 , 317-348. [CrossRef]

37. Martyushev, L.M.; Seleznev, V.D. Maximum entropy production principle in physics, chemistry and biology. Phys. Rep. 2006, 426, 1-45. [CrossRef]

38. Maysman, F.J.R.; Bruers, S. A thermodynamic perspective on food webs: Quantifying entropy production within detrital-based ecosystems. J. Theor. Biol. 2007, 249, 124-139. [CrossRef]

39. Kleidon, A. Nonequilibrium thermodynamics and maximum entropy production in the Earth system. Naturwissenschaften 2009, 96, 653-677. [CrossRef] 
40. Niven, R.K. Steady state of a dissipative flow-controlled system and the maximuentropy production principle. Phys. Rev. E 2009, 80, 021113. [CrossRef]

41. Ross, J.; Corlan, A.D.; Muller, S.C. Proposed Principle of Maximum Local Entropy Production. J. Phys. Chem. B 2012, 116, 7858-7865. [CrossRef]

(c)

(C) 2020 by the authors. Licensee MDPI, Basel, Switzerland. This article is an open access article distributed under the terms and conditions of the Creative Commons Attribution (CC BY) license (http://creativecommons.org/licenses/by/4.0/). 